\title{
Effects of Habitat and Human Activities on Species Richness and Assemblages of Staphylinidae (Coleoptera) in the Baltic Sea Coast
}

\author{
Ulrich Irmler \\ Department of Applied Ecology, Institute for Ecosystem Research, Christian Albrecht University, 24098 Kiel, Germany \\ Correspondence should be addressed to Ulrich Irmler, uirmler@ecology.uni-kiel.de
}

Received 15 June 2011; Accepted 6 August 2011

Academic Editor: Panagiotis Milonas

Copyright () 2012 Ulrich Irmler. This is an open access article distributed under the Creative Commons Attribution License, which permits unrestricted use, distribution, and reproduction in any medium, provided the original work is properly cited.

In 2009, the staphylind fauna was studied in six habitats of the Baltic Sea coast of Schleswig-Holstein (northern Germany). The following habitats lagoon, sandy beach, shingle beach, primary dune, wooded cliff, and woodless cliff were significantly separated by their species composition. Vegetation and soil moisture were the most important factors separating the assemblages. Lagoons exhibited the most species-rich habitat. Sandy beaches provided the highest number of endangered species. Both sandy beaches and woodless cliffs showed the highest number of exclusive species. A loss of species was determined in the gradient from sandy to shingle beaches. Few species preferred shingle beaches; abundance of Cafius xantholoma increased with the increasing amount of shingle. More species preferred the sandy conditions, for example, Polystomota grisea, P. punctatella, and Phytosus spinifer. Anotylus insecatus and Bledius defensus require distinct mixtures of sand and silt on woodless cliffs. Tourist impact on sandy beaches accounts for approximately $50 \%$ loss of species.

\section{Introduction}

In general, staphylind beetles are rarely taken into consideration in ecological investigations $[1,2]$. This is due to the fact that the identification is difficult, and little information is available on species ecology. Moreover, in several investigations, the differentiated assemblages corresponded only weakly to environmental parameters $[3,4]$. Nevertheless, Staphylinidae are one of the most species-rich coleopteran families and, thus, might give more information about biodiversity than any other arthropod group. This is particularly relevant as many species are associated with other animals, for example, with birds, mammals, or as parasitoids of flies. Staphylinid diversity not only informs about the abiotic heterogeneity of the habitat, but also about the heterogeneity produced by animal species.

Very few studies are available concerning the biodiversity of coastal habitats along the Baltic Sea. More is needed since activity by tourists on beaches has increased dramatically in the last decades [5] and coastal habitats are included in the list of endangered habitats in the Fauna-FloraHabitat (FFH) directive of the European Union (EU). Coastal lagoons are listed in a priority class [6]. Most information exists concerning salt marshes which also include studies on Staphylinidae [7-9].

Even though the fauna of beaches is well known in general in many European countries (e.g., $[10,11])$, there is very little information about the fauna of other coastal habitats and the influence of environmental parameters from the coasts of the Baltic Sea. In particular, little knowledge is available about sandy beaches and cliffs. In contrast to tidal coasts, the Baltic Sea coast has little or no tides. This is reflected by a wide distribution of terrestrial organisms towards the sea line.

Thus, the following study mainly focuses on sandy and shingle beaches and cliffs. In the present study, the relationships between staphylinid assemblages and habitat factors, the relationships between individual species and soil factors, and the effect of tourism on species richness are highlighted. We address the following questions in this study: (i) which environmental parameters control the composition of staphylinid assemblages at the coast? (ii) are soil parameters responsible for the occurrence of different 
staphylinid species? and (iii) where are the most species-rich coastal habitats and are they influenced by beach tourism?

\section{Sites and Methods}

The investigation was performed in 2009, from April 9 to August 20 at nine locations along the Baltic Sea coast in Schleswig-Holstein, northern Germany, having an extremely low tidal range of less than $10 \mathrm{~cm}$ (Figure 1). At these 9 locations, different sites were selected representing the following habitat types: woodless and wooded cliffs, sandy and shingle beaches, primary dunes, and lagoons (Table 1). Six sandy beaches were selected in contrast to three or four sites of the other habitat types since beaches were the focus of the investigation. Two of the six beaches were open to tourists. Staphylinidae were collected by means of pitfall traps. Up to eight replicate pitfall traps were installed at each site to compensate loss by tourist damage. Only four pitfall traps were included in the final analysis in order to have equal numbers of traps for the different sites. Pitfall traps with an opening of $5.6 \mathrm{~cm}$ diameter were filled with $10 \%$ vinegar and a tension-reducing agent and covered by a transparent shelter to shield against direct precipitation.

To compare the environmental conditions between the habitat types, the following environmental parameters were determined: soil moisture by difference between wet weight and dry weight of soil as mean of 11 sampling intervals; $\mathrm{pH}$ in deionised water using a WTW pH-Meter; organic matter after combustion of a dried soil sample; shingle content by sieving a larger soil sample in the field; sand content by sieving using a $0.063 \mathrm{~mm}$ sieve after oxidising the organic matter by $\mathrm{H}_{2} \mathrm{O}_{2}$; finer silt and clay material was determined by subtracting sand content [12].

The statistical analysis was performed using the program STATISTICA [13]. Data were tested according to parametric or nonparametric distribution using the KolmogorovSmirnov test. The data of environmental factors of assemblages and species richness (normal distribution) were compared using ANOVA with subsequent LSD post hoc test. Differences between two habitats were tested by $U$ test or $t$-test, correlations by Pearson correlation. To get an idea of total species richness in the habitats, Jackknife II species richness was calculated using the program PAST version 2.04 [14]. The second order of Jackknife estimator seems to be most accurate to estimate total species richness [15]. Detrended correspondence analysis (DCA) and canonical correspondence analysis (CCA) were executed using the program CANOCO [16]. According to ter Braak [17], a distinct ordination of assemblages can be expected at eigenvalues higher than 0.5. Monte Carlo Permutation test was performed to find the significance of environmental parameters.

In the beach habitats, sand and shingle contents were closely correlated: sand content $=97+0.96^{*}$ shingle content $(r=0.99 ; P<0.001)$. Therefore, either sand content or shingle content was used to analyse the occurrence of species in the sand-shingle gradient. For some comparisons, the sandshingle gradient was subdivided into the following three classes: shingle: $>70 \%$ shingle and $<30 \%$ sand; medium: $10-60 \%$ shingle and $40-90 \%$ sand; sand: $>90 \%$ sand and $<10 \%$ shingle. To analyse the abundance in these gradients, Kruskal-Wallis ANOVA was used with subsequent $U$ test and Bonferroni correction using the program STATISTICA. The status of endangered species refers to the red list of SchleswigHolstein [18].

\section{Results}

3.1. Environmental Parameters. The highest soil moisture contents were found at the lagoons and wooded cliffs, while all beach habitats have very low soil moistures without significant differences (Table 2). Sand content was high at the sandy beaches and the primary dunes and lowest at the wooded cliffs and the shingle beaches. The shingle content differs between shingle beach with $77 \%$ on average and all other habitats. Soil $\mathrm{pH}$ was on a high level in a narrow range. Nevertheless, significant differences were also found for this parameter. The highest soil $\mathrm{pH}$ was found at the woodless cliffs and at the shingle beaches, and the lowest $\mathrm{pH}$ at the primary dunes. Thus, sandy beaches and primary dunes that show no differences in all other parameters vary significantly in their soil $\mathrm{pH}$. Organic material was low in all habitats, but highest in wooded cliffs and lagoons. Overall, each habitat type reflected a specific combination of soil parameters. They could be significantly separated by at least one of the measured parameters.

3.2. Species Composition. From a total of 4324 specimens collected, 165 species have been identified. The highest number of species was found at lagoons, and the lowest number of species on shingle beaches (Table 3). Jackknife species richness was also highest at lagoons and lowest at shingle beaches. The highest number of endangered species was found on sandy beaches. Only primary dunes have no endangered species.

In correspondence to the environmental differences between the habitats, the detrended correspondence analysis revealed assemblages of rove beetle that are clearly separated by their habitat characterisation (Figure 2). Only the shingle beach at Weissenhaus was attributed to the sandy beaches and not to the other three shingle beaches. According to the canonical correspondence analysis, 3 of the 6 factors have a significant effect on the species composition of the assemblages and account for $76 \%$ of the total variance. The most important factor was soil moisture $(F=3.6)$ which accounted for $34 \%$ of the total variance. Wooded or nonwooded situation accounted for $23 \%(F=2.8)$, and soil $\mathrm{pH}$ accounted for $21 \%(F=2.7)$. Neither sand content nor shingle content nor content of organic matter were significant for the separation of the assemblages.

The composition of dominant species in the 6 habitat types shows that several species are widely distributed along the different habitat types (Table 4 ). The widely distributed Aleochara sparsa was found highly dominant in the cliff habitats. While several species frequently found in SchleswigHolstein revealed highest dominance in the wooded cliffs, 


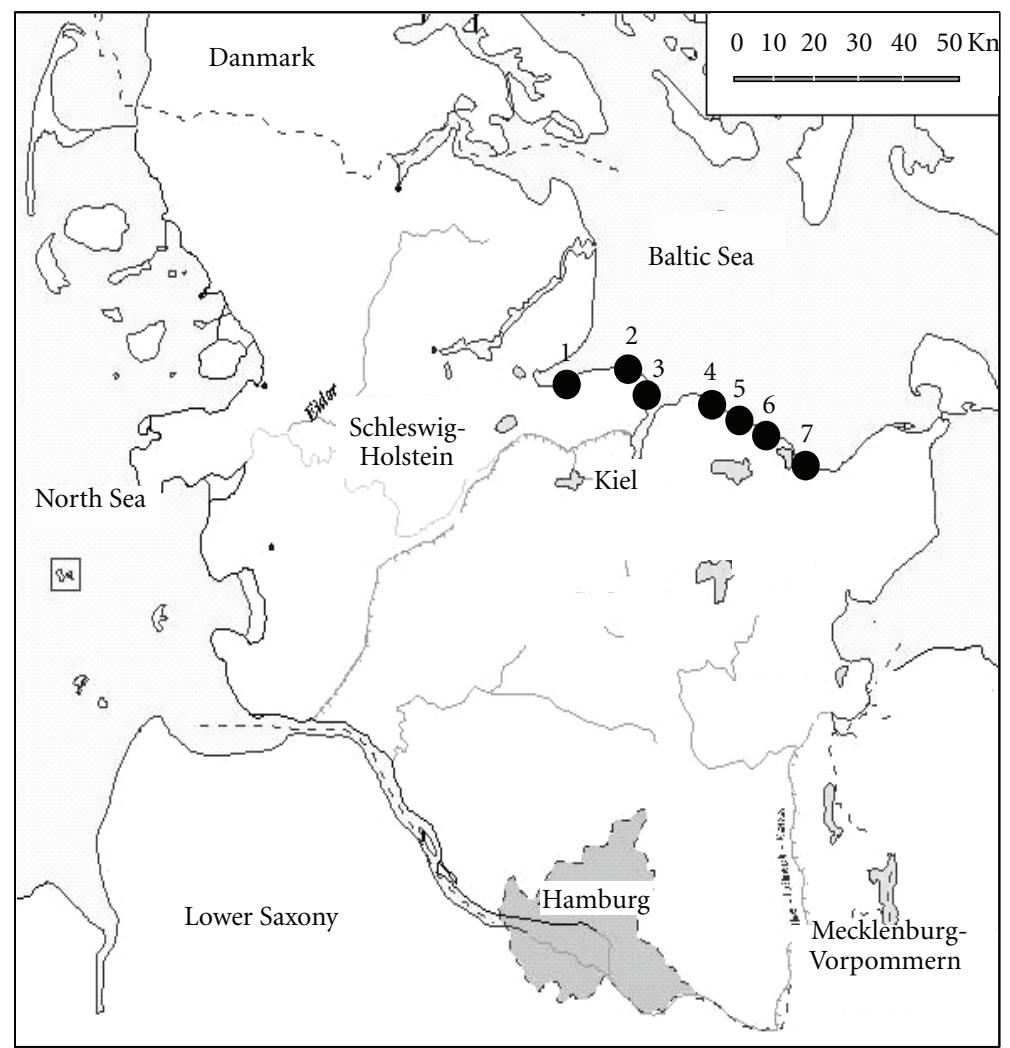

Figure 1: Investigated locations at the Baltic Sea coast: Lindhöft (1), Dänisch-Nienhof (2), Stohl (3), Stakendorf and Hohenfelde (4), Hubertsberg (5), Behrensdorf and Lippe (6), and Weißenhaus (7).

TABLE 1: Habitats at the investigated locations and abbreviations of locations and habitats; $\mathrm{x}$ indicates a number of 4 replicate pitfall traps.

\begin{tabular}{|c|c|c|c|c|c|c|c|}
\hline $\begin{array}{l}\text { Location/Habitat } \\
\text { (abbreviation) }\end{array}$ & $\begin{array}{l}\text { Tourism on } \\
\text { sandy beach }\end{array}$ & Lagoon (L) & $\begin{array}{c}\text { Sandy beach } \\
\text { (SB) }\end{array}$ & $\begin{array}{c}\text { Shingle beach } \\
\text { (GB) }\end{array}$ & $\begin{array}{c}\text { Primary dune } \\
(\mathrm{PD})\end{array}$ & $\begin{array}{l}\text { Woodless cliff } \\
(\mathrm{Wl})\end{array}$ & $\begin{array}{l}\text { Wooded cliff } \\
(\mathrm{Wc})\end{array}$ \\
\hline Behrensdorf (BD) & Closed & $\mathrm{x}$ & $\mathrm{x}$ & - & - & - & - \\
\hline Dänisch-Nienhof (DN) & - & - & - & $\mathrm{x}$ & - & $\mathrm{x}$ & $\mathrm{x}$ \\
\hline Hubertsberg (HB) & - & - & - & $\mathrm{x}$ & - & $\mathrm{x}$ & - \\
\hline Hohenfelde (HF) & Open & $\mathrm{x}$ & $\mathrm{x}$ & - & $\mathrm{x}$ & - & - \\
\hline Lippe (KB) & Closed & - & $\mathrm{x}$ & - & $\mathrm{x}$ & - & - \\
\hline Lindhöft (LH) & Open & - & $\mathrm{x}$ & - & - & - & $\mathrm{x}$ \\
\hline Stakendorf (SD) & Closed & $\mathrm{x}$ & $\mathrm{x}$ & - & $\mathrm{x}$ & - & - \\
\hline Stohl (ST) & - & - & - & $\mathrm{x}$ & - & $\mathrm{x}$ & - \\
\hline Weißenhaus (WH) & Closed & - & $\mathrm{x}$ & $\mathrm{x}$ & $\mathrm{x}$ & $\mathrm{x}$ & $\mathrm{x}$ \\
\hline
\end{tabular}

TABLE 2: Mean values of environmental parameters for the differentiated staphylinid assemblages; different exponents indicate significant differences by ANOVA and consecutive LSD test.

\begin{tabular}{lcccccc}
\hline Parameter & Wooded cliffs & Woodless cliffs & Lagoon & Sandy beach & Primary dune & Shingle beach \\
\hline Soil moisture (\%) & ${ }^{\mathrm{a}} 18 \pm 3.0$ & ${ }^{\mathrm{b}} 8 \pm 1.1$ & ${ }^{\mathrm{a}} 31 \pm 5.9$ & ${ }^{\mathrm{c}} 1 \pm 0.5$ & ${ }^{\mathrm{c}} 1 \pm 0.5$ & ${ }^{\mathrm{c}} 1 \pm 0.6$ \\
Wood & yes & no & no & no & no & no \\
Sand content (\%) & ${ }^{\mathrm{b}} 36 \pm 6$ & ${ }^{\mathrm{c}} 46 \pm 3$ & ${ }^{\mathrm{c}} 51 \pm 16$ & ${ }^{\mathrm{a}} 89 \pm 9$ & ${ }^{\mathrm{a}} 92 \pm 9$ & ${ }^{\mathrm{b}} 22 \pm 21$ \\
Shingle content (\%) & $\mathrm{b}^{\mathrm{b}} \pm 2$ & $\mathrm{~b} 8 \pm 2$ & ${ }^{\mathrm{b}} 21 \pm 20$ & ${ }^{\mathrm{b}} 8 \pm 10$ & ${ }^{\mathrm{b}} 6 \pm 8$ & ${ }^{\mathrm{a}} 77 \pm 20$ \\
Soil pH & ${ }^{\mathrm{b}} 7.4 \pm 0.1$ & ${ }^{\mathrm{a}} 7.9 \pm 0.2$ & ${ }^{\mathrm{b}} 7.5 \pm 0.2$ & ${ }^{\mathrm{b}} 7.3 \pm 0.2$ & ${ }^{\mathrm{c}} 7.2 \pm 0.1$ & ${ }^{\mathrm{a}} 7.9 \pm 0.3$ \\
Organic content of soil (\%) & ${ }^{\mathrm{a}} 6.6 \pm 2.6$ & ${ }^{\mathrm{b}} 1.1 \pm 0.1$ & ${ }^{\mathrm{a}} 5.6 \pm 0.9$ & ${ }^{\mathrm{b}} 0.2 \pm 0.2$ & ${ }^{\mathrm{b}} 1.4 \pm 2.2$ & ${ }^{\mathrm{b}} 0.3 \pm 0.1$ \\
\hline
\end{tabular}


TABLE 3: Species richness in the investigated habitats and Jackknife II species richness; RL species richness of endangered species (only status 1 and 2).

\begin{tabular}{|c|c|c|c|c|c|c|c|c|c|c|c|c|c|}
\hline \multirow{2}{*}{ Habitat type } & \multirow[b]{2}{*}{$\mathrm{BD}$} & \multicolumn{7}{|c|}{ Localities } & \multicolumn{5}{|c|}{ Species richness } \\
\hline & & $\mathrm{DN}$ & $\mathrm{HF}$ & $\mathrm{HB}$ & $\mathrm{KB}$ & LH & SD & ST & WH & Total & Per trap & Jackknife II & RL \\
\hline Lagoon & 37 & - & 47 & - & - & - & 51 & - & - & 85 & $13.9 \pm 2.5$ & $154 \pm 6.5$ & 2 \\
\hline Sandy beach & 36 & - & 10 & - & 27 & 18 & 21 & - & 22 & 70 & $10.0 \pm 4.2$ & $116 \pm 6.6$ & $\underline{5}$ \\
\hline Shingle beach & - & 9 & - & 21 & - & - & - & 13 & 5 & 33 & $4.4 \pm 3.3$ & $61 \pm 5.5$ & 2 \\
\hline Primary dune & - & - & 21 & - & 17 & - & 28 & - & 28 & 75 & $9.4 \pm 4.0$ & $88 \pm 5.3$ & 0 \\
\hline Woodless cliff & - & 18 & - & 38 & - & - & - & 27 & 20 & 58 & $10.7 \pm 3.5$ & $99 \pm 7.1$ & 1 \\
\hline Wooded cliff & - & 22 & - & - & - & 26 & - & - & 19 & 43 & $8.7 \pm 3.4$ & $72 \pm 4.8$ & 1 \\
\hline
\end{tabular}

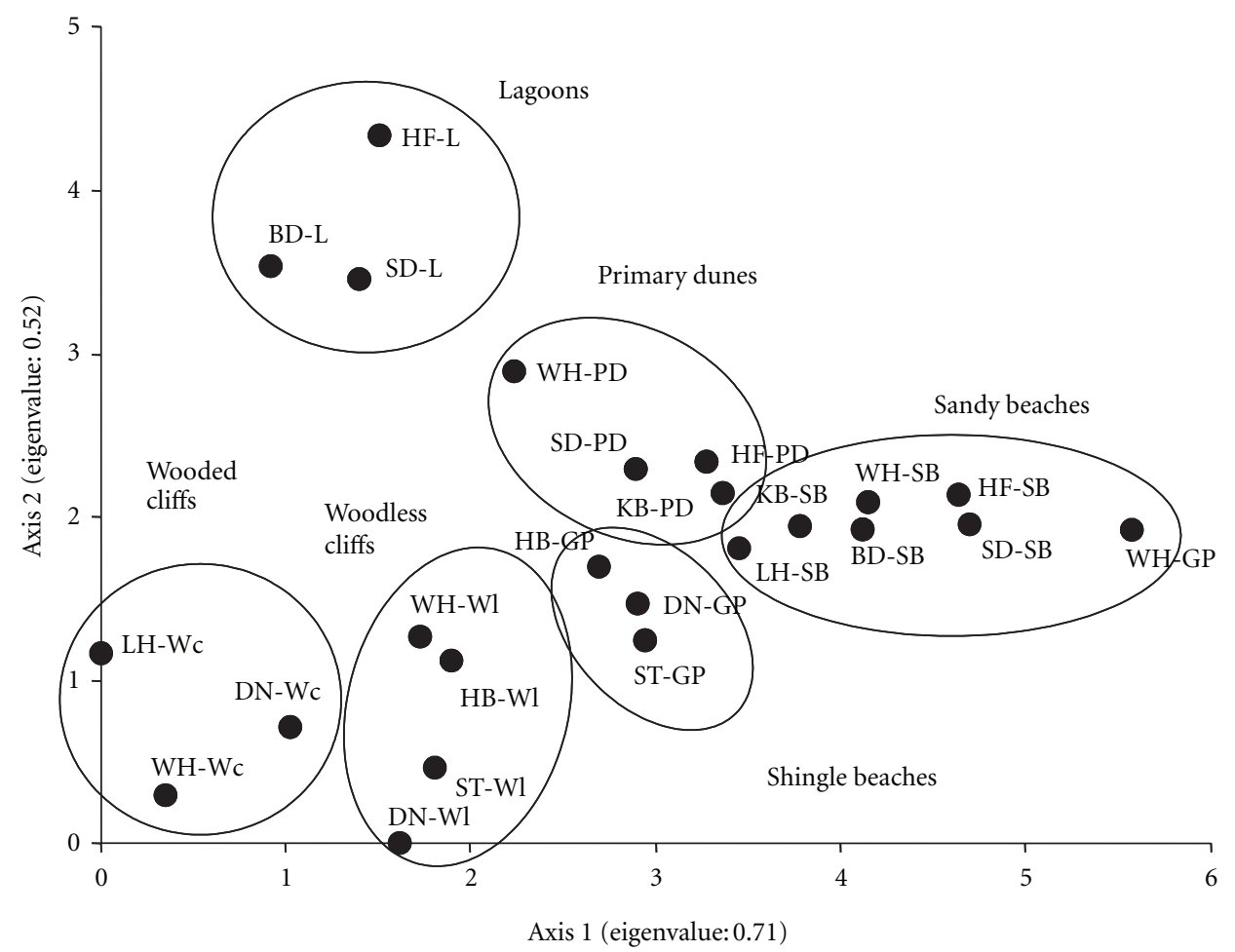

Figure 2: Results of the detrended correspondence analysis showing the separation of the 6 habitat types along the first two axes.

a higher number of specialised species were found in the woodless cliffs. In particular, rare species of bare soils, for example, Stenus fossulatus, Bledius erraticus, Bledius defensus, and Anotylus insecatus, were almost exclusively recorded there. Lagoons as wooded cliffs have no remarkable species that are restricted to the specific lagoon situation. All dominant species are frequently found also on mainland lake shores. Primary dunes showed only one specific species, that is Ocypus brunnipes, in contrast to the two beach habitats, where a number of 8 species can be regarded as typically independent from the soil situation. Shingle beaches thus seem to have no specific species in comparison to sandy beaches. In contrast, 3 species, that is, Phytosus balticus, Bledius subniger, and Atheta vestita, seem to prefer the sandy beaches.
3.3. Relationships between Individual Species and Environmental Parameters. Although the differences between shingle beaches, sandy beaches, and primary dunes are minor in rove beetle assemblages, individual species show significant differences concerning their occurrence. Cafius xantholoma only inhabits the beach habitats and was never found on the adjacent primary dunes (ANOVA: beach versus primary dune: $F=6.7, P<0.01$ ). Along sand gradient of beaches, the species significantly prefers the shingle beaches; a significant linear increase along the sand-shingle gradient was found (abundance $=0.02 \cdot$ shingle content $+0.36, r=0.66, P=$ $0.04)$. A similar restriction to the beach habitats was found for Polystomota grisea (ANOVA: beach versus primary dune: $F=17.2, P<0.001$ ) and Polystomota punctatella (ANOVA: beach versus primary dune: $F=10.9, P<0.002$ ). In contrast 


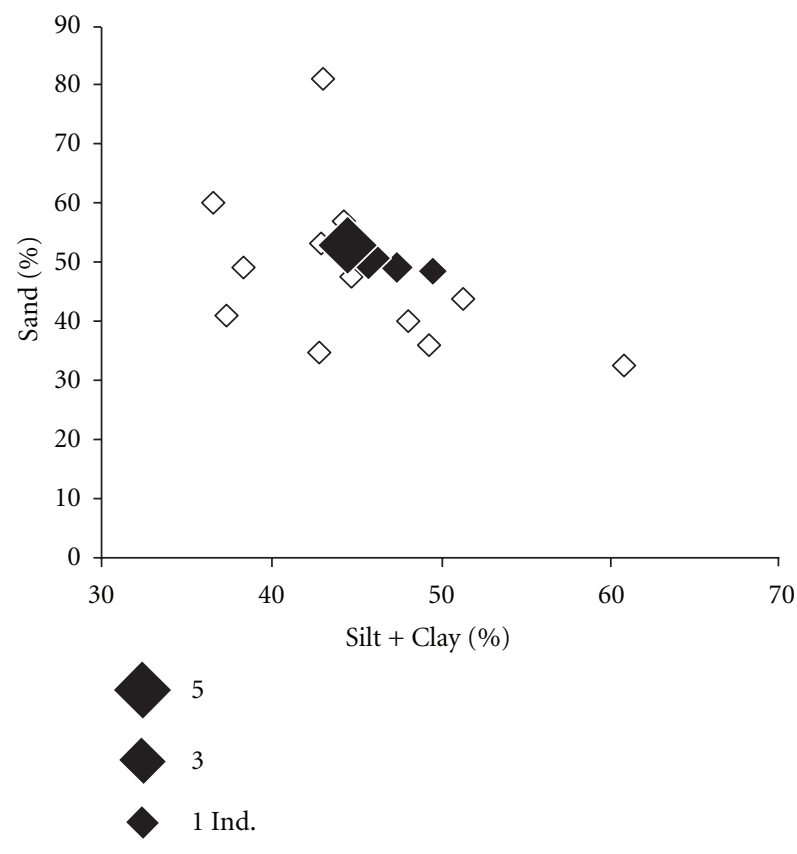

(a)
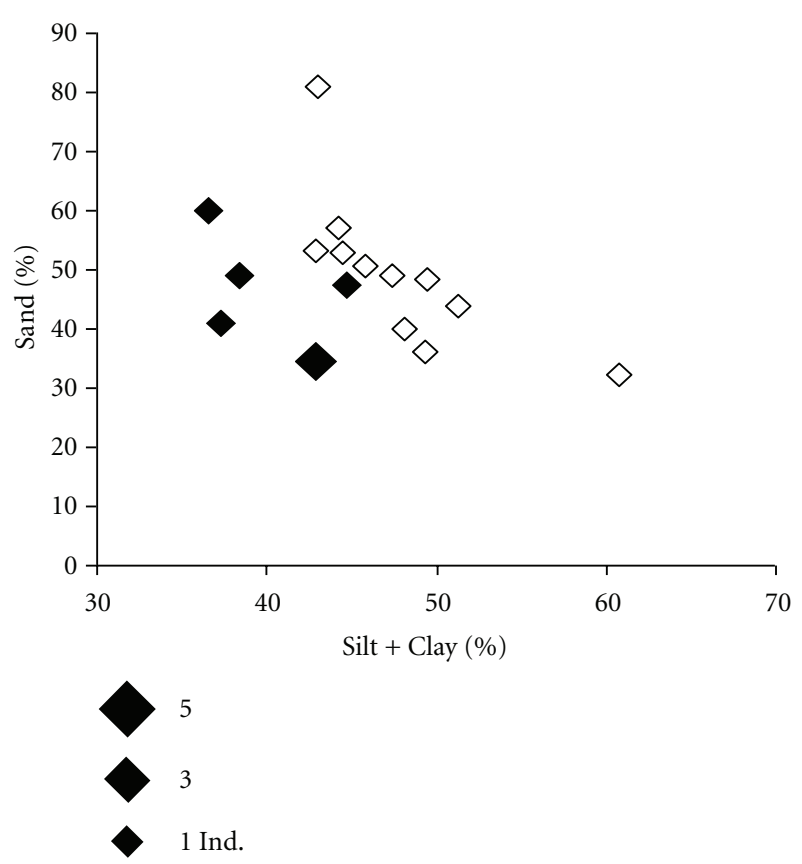

1 Ind.

FIGURE 3: Occurrence of Bledius defensus (a) and Anotylus insecatus (b) in the sand-silt/clay relationship of woodless cliffs.

to C. xantholoma, both species significantly preferred the sandy beaches: $P$. grisea abundance $=0.03 \cdot$ sand content $0.27, r=0.73, P=0.02$; $P$. Punctatella abundance $=$ 0.02 sand content $+0.19, r=0.67, P=0.03$. Furthermore, Phytosus spinifer also occurred only in the beach habitats and preferred significantly the beaches of the sand class (Kruskal-Wallis ANOVA using the three classes: $\mathrm{Chi}^{2}=7.8$, $P=0.02$ ). The species was absent on beaches with a shingle content greater than $10 \%$ and on beaches with a sand content lower than $80 \%$. In contrast to the four species restricted to beaches, Aleochara bipustulata was found on sandy beaches and primary dunes in similar abundance (ANOVA: $F=2.1$, $P=0.1$, not significant). However, the species significantly preferred the sandy habitats. Using all sites of beaches and primary dunes for the correlation between abundance and sand content, abundance increases with an increase in sand content of the habitat (abundance $=0.53 \cdot$ sand content 6.18, $r=0.67, P=0.006$ ).

For three species of the woodless cliffs, preference for specific mixtures of silt/clay and sand is assumed in regard to their occurrence in the silt/clay-sand relationship (Figure 3). Anotylus insecatus was mainly found on woodless cliffs between $30 \%$ and $40 \%$ silt/clay content, while it was absent from cliffs with silt/clay contents higher than 50\% (KurskalWallis ANOVA: $\left.\mathrm{Chi}^{2}=8.8, P=0.01\right)$. Bledius defensus was found in a silt/clay range between $40 \%$ and $50 \%$ (KurskalWallis ANOVA: $\mathrm{Chi}^{2}=5.8, P=0.04$ ). The species was not found at cliffs with silt/clay contents lower than $40 \%$. Only 1 specimen was found at cliffs with silt/clay contents higher than $50 \%$. The species seems to occur in a very restricted range having a sand content between $45 \%$ and $50 \%$ and silt/clay content between $35 \%$ and 52\%. Another typical species of woodless cliffs, that is Stenus fossulatus, showed no significant preference of specific sand-silt/clay mixtures. It occurred in woodless cliffs with a range of sand content between $42 \%$ and $50 \%$ and a wide range of silt/clay content between $36 \%$ and $81 \%$. However, soil moisture on woodless cliffs was important. Abundance increased with increasing soil moisture (abundance $=0.39 \cdot$ soil moisture $-2.21, r=$ $0.96, P=0.03)$.

3.4. Effect of Tourism. Sandy beaches closed to tourists have significant higher species richness than beaches open to tourism (Table 5). The closed beaches revealed nearly twice as many species as the beaches open to tourists. No species was found exclusively on beaches open to tourists. Thus, half of the species of closed beaches are absent from open beaches. The difference between the two beach types is still more obvious if Jackknife II species richness is considered. Three species of the aleocharinae subfamily were abundant enough to analyse their occurrence in the two beach types. Both Aleochara bipustulata and Polystomota grisea showed significantly higher abundance on the beaches closed to tourists. A significant difference between both beach types concerning their sand content was not found ( $U$ test: $Z=$ $8.8, P=0.06$ ). Thus, the differences found can be referred to the tourist impact. Only in Polystomota punctatella was the difference not significant.

\section{Discussion}

Unfortunately, no other studies concerning staphylinids from beach habitats considering environmental parameters are available for comparison. Rose [3] investigated 
TABLE 4: Dominance of species that contribute to the species characterisation of the habitats.

\begin{tabular}{|c|c|c|c|c|c|c|}
\hline Species & Wooded cliff & Woodless cliff & Lagoon & Primary dune & Shingle beach & Sandy beach \\
\hline Tasgius morsitans & 5.0 & 0.4 & 0.1 & 0.2 & - & - \\
\hline Omalium rivulare & 4.3 & - & 0.6 & - & 0.8 & 0.1 \\
\hline Atheta crassicornis & 3.2 & 0.1 & 0.8 & 0.2 & 0.8 & - \\
\hline Lathrimaeum unicolor & 2.8 & 0.4 & 0.2 & 0.2 & - & 0.1 \\
\hline Quedius fuliginosus & 2.8 & 0.3 & 1.0 & - & - & - \\
\hline Atheta atramentaria & 2.1 & 0.4 & 0.1 & 0.4 & - & 2.2 \\
\hline Aleochara sparsa & 50.2 & 13.5 & 0.8 & 1.8 & 2.3 & 1.7 \\
\hline Stenus fossulatus & 0.7 & 2.2 & - & - & - & - \\
\hline Tachyporus dispar & 1.1 & 5.4 & - & 0.3 & - & 0.1 \\
\hline Bledius erraticus & - & 1.6 & - & - & - & 0.1 \\
\hline Bledius defensus & - & 1.3 & - & - & - & 0.1 \\
\hline Tasgius winkleri & - & 0.9 & 0.1 & - & - & - \\
\hline Anotylus insecatus & - & 0.9 & - & - & - & - \\
\hline Tachyporus nitidulus & 1.8 & 27.1 & 0.4 & 0.1 & 12.5 & 0.1 \\
\hline Drusilla canaliculata & 1.1 & 24.6 & 18.3 & 13.2 & 16.4 & 1.1 \\
\hline Stenus pallipes & - & - & 3.3 & - & - & - \\
\hline Stenus juno & 0.4 & - & 4.1 & 0.2 & - & - \\
\hline Atheta graminicola & - & 0.3 & 8.9 & - & - & 0.1 \\
\hline Stenus canaliculatus & - & 0.4 & 3.3 & - & - & - \\
\hline Pachnida nigella & - & - & 2.6 & - & - & 0.1 \\
\hline Ocypus brunnipes & - & - & 0.2 & 1.6 & & 0.4 \\
\hline Aleochara bipustulata & 0.7 & 2.8 & 2.9 & 72.5 & 19.5 & 56.7 \\
\hline Polystomota grisea & - & - & 0.1 & - & 7.0 & 5.4 \\
\hline Polystomota punctatella & - & - & - & - & 1.6 & 5.1 \\
\hline Cafius xantholoma & - & - & - & - & 7.8 & 4.7 \\
\hline Omalium riparium & - & - & - & 0.1 & 0.8 & 0.8 \\
\hline Phytosus spinifer & - & - & 一 & - & 0.2 & 0.8 \\
\hline Phytosus balticus & - & - & - & - & - & 0.3 \\
\hline Bledius subniger & - & - & - & 0.2 & - & 0.8 \\
\hline Atheta vestita & - & - & 0.1 & 0.3 & 一 & 2.2 \\
\hline
\end{tabular}

TABLE 5: Species richness and median abundance of three species in four sandy beaches closed to tourists and two beaches open to tourists with results of $t$-test or $U$ test; significant differences are underlined.

\begin{tabular}{|c|c|c|c|c|c|c|c|c|}
\hline \multirow{2}{*}{$\begin{array}{l}\text { Situation of } \\
\text { beach }\end{array}$} & \multirow[t]{2}{*}{$n$ traps } & \multicolumn{4}{|c|}{ Species richness } & \multirow{2}{*}{$\begin{array}{c}\text { Aleochara bipustulata } \\
\text { Median }\end{array}$} & \multirow{2}{*}{$\begin{array}{c}\text { Polystomota grisea } \\
\text { Median }\end{array}$} & \multirow{2}{*}{$\begin{array}{c}\text { Polystomota punctatella } \\
\text { Median }\end{array}$} \\
\hline & & Species per trap & S.D & Jackknife II & S.D. & & & \\
\hline Closed & 16 & $\underline{11.6}$ & 4.2 & 109 & 6.3 & $\underline{28.0}$ & $\underline{2.0}$ & 1.0 \\
\hline Open & 8 & 6.8 & 1.8 & 56 & 3.4 & 2.5 & 0.0 & 0.0 \\
\hline$t / Z$ & & 3.1 & & & & 3.7 & 2.5 & 0.6 \\
\hline$P$ & & 0.005 & & $<0.001$ & & $<0.001$ & 0.01 & 0.5 \\
\hline
\end{tabular}

the staphylinid fauna of three North Sea islands of Lower Saxony, but beaches were not included. In his study of dunes, salt marshes, and bushy vegetations, the eigenvalues of the first axes ranged between 0.26 and 0.41 . This is remarkably lower than the value of 0.71 in the present study, which indicates a greater dissimilarity between the habitats of the Baltic Sea coast. The higher similarity of the species compositions on the North Sea islands might be referred to the island situation, where a lower diversity might have developed than in mainland habitats. However, the high species richness on the three North Sea islands, ranging between 227 and 269 species, does not support this hypothesis; the number of species was higher than the 165 species found in the present study. It is more likely that the similar sandy soil conditions of the three North Sea islands are responsible for the higher similarity of the rove beetle assemblages. In the present study, clay, sand, and shingle soils were included. Similar results concerning environmental parameters were found 
TABLE 6: List of staphylinid species (total number of individuals) at the investigated sites.

\begin{tabular}{|c|c|c|c|c|c|c|c|c|}
\hline Species & Lindhof & Dänisch-Nienhof & Stohl & Stakendorf & Hohenfelde & Hubertsberg & Behrensdorf & Weißenhaus \\
\hline Acidota crenata & - & - & - & - & - & - & 1 & - \\
\hline Aleochara bilineata & - & - & 1 & - & - & 3 & 5 & - \\
\hline Aleochara binotata & - & - & - & - & 1 & - & 4 & - \\
\hline Aleochara bipustulata & - & 12 & 5 & 141 & 363 & 29 & 878 & 221 \\
\hline Aleochara brevipennis & - & - & - & - & - & - & 1 & - \\
\hline Aleochara sparsa & 26 & 104 & 28 & 14 & 1 & 22 & 25 & 65 \\
\hline Aleochara verna & - & - & - & - & 1 & - & 3 & 4 \\
\hline Aloconota gregaria & 1 & 2 & 1 & 8 & 7 & 3 & 11 & 13 \\
\hline Amischa analis & - & - & - & 4 & 2 & 2 & 10 & 9 \\
\hline Amischa decipiens & - & - & - & - & - & - & 2 & - \\
\hline Amischa soror & - & - & 1 & 1 & 1 & 3 & 2 & - \\
\hline Anotylus insecatus & - & 2 & - & - & - & 3 & - & 1 \\
\hline Anotylus rugosus & 2 & 1 & 1 & 28 & 23 & 2 & 15 & 3 \\
\hline Anotylus sculpturatus & 1 & 1 & 1 & 1 & - & 3 & - & 3 \\
\hline Anotylus tetracarinatus & - & 3 & - & 2 & 11 & - & 2 & - \\
\hline Atheta amicula & - & - & 1 & 1 & - & 1 & 1 & - \\
\hline Atheta atramentaria & 4 & 4 & - & 5 & 8 & 1 & 18 & 3 \\
\hline Atheta cauta & - & - & - & 1 & - & - & 1 & - \\
\hline Atheta celata & - & - & - & 1 & - & 1 & 1 & - \\
\hline Atheta crassicornis & 7 & 2 & - & 5 & 2 & 2 & - & 2 \\
\hline Atheta elongatula & - & - & - & - & 1 & - & - & - \\
\hline Atheta fungi & 1 & 8 & - & 9 & 136 & 1 & 12 & 19 \\
\hline Atheta gagatina & - & - & - & - & - & - & - & 1 \\
\hline Atheta graminicola & - & - & - & 11 & 52 & 2 & 19 & - \\
\hline Atheta ischnocera & - & - & - & - & - & - & 1 & - \\
\hline Atheta laticollis & - & - & - & 5 & - & - & - & - \\
\hline Atheta liliputana & - & - & - & - & - & - & 1 & 1 \\
\hline Atheta luteipes & - & - & - & - & 4 & - & 1 & - \\
\hline Atheta marcida & - & - & - & - & - & - & 2 & - \\
\hline Atheta melanaria & - & - & - & - & - & - & 1 & - \\
\hline Atheta nigricornis & 1 & 2 & 2 & - & - & - & - & 1 \\
\hline Atheta oblita & - & - & - & 1 & - & - & 1 & 1 \\
\hline Atheta palustris & - & - & 1 & - & - & 8 & - & 1 \\
\hline Atheta sodalis & 2 & - & - & - & - & 1 & - & - \\
\hline Atheta sp. & 3 & - & - & - & - & - & - & - \\
\hline Atheta triangulum & 1 & 1 & - & - & 2 & 6 & 2 & 2 \\
\hline Atheta vestita & 3 & - & - & 13 & 1 & - & 4 & 1 \\
\hline Atheta volans & 1 & - & 1 & 1 & 1 & 1 & 1 & - \\
\hline Bledius defensus & 1 & - & 1 & - & - & - & - & 8 \\
\hline Bledius erraticus & 1 & 11 & - & 1 & 1 & - & - & 1 \\
\hline Bledius opacus & - & - & - & 1 & - & - & - & - \\
\hline Bledius pallipes & - & 1 & - & - & - & - & - & - \\
\hline Bledius subniger & - & - & - & 7 & - & - & 1 & - \\
\hline Brundinia marina & - & - & - & - & - & 1 & 1 & 2 \\
\hline Brundinia meridionalis & - & - & - & - & - & - & 1 & - \\
\hline Cafius xantholoma & - & 4 & 3 & 7 & - & 3 & 15 & 14 \\
\hline Callicerus obscurus & - & 1 & - & - & - & - & - & - \\
\hline Calodera aethiops & - & - & - & 2 & 2 & - & 3 & - \\
\hline Carpelimus corticinus & - & - & 1 & 5 & 13 & 3 & 7 & - \\
\hline Carpelimus elongatus & 1 & - & - & - & - & - & - & \\
\hline
\end{tabular}


Table 6: Continued.

\begin{tabular}{|c|c|c|c|c|c|c|c|c|}
\hline Species & Lindhof & Dänisch-Nienhof & Stohl & Stakendorf & Hohenfelde & Hubertsberg & Behrensdorf & Weißenhaus \\
\hline Carpelimus foveolatus & - & - & - & 1 & - & - & - & - \\
\hline Carpelimus gracilis & - & - & - & - & - & - & 1 & - \\
\hline Carpelimus rivularis & - & - & - & - & - & - & - & 1 \\
\hline Cordalia obscura & - & - & - & - & - & - & - & 1 \\
\hline Cryptobium collare & - & - & - & - & 1 & - & - & - \\
\hline Cypha discoideus & - & - & - & - & 1 & - & - & - \\
\hline Dacrila fallax & - & - & - & 1 & - & - & 7 & - \\
\hline Dinaraea angustula & - & 5 & 3 & 5 & 6 & 13 & 5 & 4 \\
\hline Drusilla canaliculata & 1 & 4 & 6 & 59 & 9 & 115 & 123 & 218 \\
\hline Euaesthetus bipunctatus & - & - & - & - & 1 & - & - & - \\
\hline Euaesthetus laeviusculus & - & - & - & - & - & - & 1 & - \\
\hline Falagria thoracica & - & - & 1 & 5 & - & 1 & - & - \\
\hline Gabrius breviventer & - & 1 & - & 2 & 8 & - & 7 & - \\
\hline Gabrius vernalis & - & - & - & - & - & - & 2 & - \\
\hline Geostiba circellaris & - & - & - & 1 & - & - & - & - \\
\hline Gnypeta carbonaria & - & - & - & - & - & - & 1 & - \\
\hline Gyrohypnus angustatus & - & - & - & - & - & 1 & - & 5 \\
\hline Habrocerus capilaricornis & - & 1 & - & - & - & - & - & - \\
\hline Halobrecta puncticeps & 2 & - & - & 6 & 5 & - & 2 & 1 \\
\hline Haploglossa nidicola & - & - & - & - & - & - & - & 1 \\
\hline Heterothops dissimilis & - & - & - & 2 & - & - & - & - \\
\hline Hydrosmecta longula & 3 & - & - & 1 & - & 2 & 1 & 1 \\
\hline Ilyobates bennetti & - & - & - & - & 1 & - & - & - \\
\hline Ischnosoma splendidus & - & - & 1 & 1 & 3 & - & 1 & - \\
\hline Lathrimaeum unicolor & 6 & - & - & 1 & - & 2 & 1 & 6 \\
\hline Lathrobium brunnipes & 1 & - & - & 1 & - & - & - & - \\
\hline Lathrobium fulvipenne & - & - & 1 & 1 & - & - & - & - \\
\hline Lathrobium longulum & - & - & - & 一 & 1 & - & - & - \\
\hline Lathrobium rufonitidum & - & - & - & 2 & - & - & - & - \\
\hline Lesteva longoelytrata & - & 3 & 10 & 3 & - & 3 & 1 & 4 \\
\hline Lesteva sicula & - & - & - & - & 1 & - & - & - \\
\hline Liogluta alpestris & - & - & - & 1 & - & - & - & 1 \\
\hline Lordithon thoracicus & - & - & - & - & - & - & 1 & - \\
\hline Myllaena intermedia & - & - & - & - & 1 & - & 1 & - \\
\hline Myllaena minuta & - & - & - & - & 2 & - & 3 & - \\
\hline Myrmeocephalus concinnus & - & - & - & - & - & 1 & - & - \\
\hline Nehemitropia lividipennis & - & - & - & - & - & - & 2 & - \\
\hline Neobisnius procerulus & - & - & - & 1 & - & - & - & - \\
\hline Ocalea picata & 4 & - & - & - & - & - & - & - \\
\hline Ocypus brunnipes & - & - & - & 2 & 19 & - & 1 & 4 \\
\hline Ocypus similis & - & - & - & 2 & 1 & - & - & - \\
\hline Omalium littorale & - & - & - & 1 & - & - & 3 & 2 \\
\hline Omalium riparium & 5 & - & 1 & 1 & - & - & 1 & 1 \\
\hline Omalium rivulare & 5 & 1 & 1 & 6 & - & - & - & 6 \\
\hline Omalium rugatum & - & - & - & - & - & - & - & 3 \\
\hline Othius punctulatus & 2 & - & - & - & - & - & - & - \\
\hline Oxypoda acuminata & - & 1 & - & 1 & - & - & - & 2 \\
\hline Oxypoda brachyptera & - & 1 & 2 & - & - & 2 & - & - \\
\hline Oxypoda brevicornis & - & - & - & - & - & - & 2 & - \\
\hline Oxypoda elongatula & - & - & - & 4 & 3 & - & 8 & - \\
\hline Oxypoda haemorrhoa & - & - & - & - & - & - & - & 1 \\
\hline
\end{tabular}


Table 6: Continued.

\begin{tabular}{|c|c|c|c|c|c|c|c|c|}
\hline Species & Lindhof & Dänisch-Nienhof & Stohl & Stakendorf & Hohenfelde & Hubertsberg & Behrensdorf & Weißenhaus \\
\hline Oxypoda procerula & - & - & - & 2 & 8 & - & 5 & - \\
\hline Oxytelus fulvipes & - & 1 & - & - & - & - & - & - \\
\hline Oxytelus sculptus & - & - & - & - & 1 & - & - & - \\
\hline Pachnida nigella & - & - & - & 18 & 6 & - & 1 & - \\
\hline Paederus riparius & - & - & - & 8 & - & - & - & - \\
\hline Philonthus cognatus & - & - & - & - & - & 1 & - & - \\
\hline Philonthus decorus & 1 & - & - & - & - & - & - & - \\
\hline Philonthus fumarius & - & - & - & 6 & - & - & - & - \\
\hline Philonthus fuscipennis & - & - & - & - & - & - & - & 1 \\
\hline Philonthus micans & - & - & - & 2 & - & - & - & - \\
\hline Philonthus quisquiliarius & - & - & - & - & - & - & 1 & - \\
\hline Phytosus balticus & - & - & - & 2 & - & - & - & - \\
\hline Phytosus spinifer & - & 1 & - & 1 & - & - & 6 & - \\
\hline Placusa depressa & - & - & - & - & - & - & 1 & - \\
\hline Placusa pumilio & - & 1 & - & - & - & - & - & - \\
\hline Plataraea brunnea & 2 & - & - & - & - & - & - & - \\
\hline Platydracus stercorarius & - & - & - & 1 & - & - & - & 1 \\
\hline Polystomota grisea & 2 & - & 9 & 21 & 1 & - & 17 & 3 \\
\hline Polystomota punctatella & 10 & 1 & 1 & 5 & - & - & 26 & - \\
\hline Quedius fuliginosus & 5 & - & 2 & 4 & 3 & - & 2 & 3 \\
\hline Quedius fumatus & 1 & - & - & - & - & - & - & - \\
\hline Quedius molochinus & - & - & 2 & - & - & - & - & - \\
\hline Quedius picipes & 1 & - & - & - & - & - & - & - \\
\hline Quedius xanthopus & 1 & - & - & - & - & - & - & - \\
\hline Rugilus rufipes & 1 & - & - & - & - & 1 & - & 2 \\
\hline Scopaeus minutus & - & - & - & - & - & 1 & - & - \\
\hline Sepedophilus marshami & - & - & - & 1 & - & - & - & - \\
\hline Stenus atratulus & - & - & - & - & 1 & - & - & - \\
\hline Stenus bimaculatus & - & 1 & - & 12 & 9 & - & 13 & - \\
\hline Stenus boops & - & - & - & - & - & - & 1 & - \\
\hline Stenus brevipennis & - & - & - & - & - & - & 1 & - \\
\hline Stenus brunnipes & - & - & - & - & - & - & 1 & - \\
\hline Stenus canaliculatus & - & - & - & 3 & - & 3 & 28 & - \\
\hline Stenus clavicornis & 1 & - & - & 6 & 6 & 2 & 6 & 2 \\
\hline Stenus formicetorum & - & - & - & 3 & 21 & - & - & - \\
\hline Stenus fossulatus & - & 2 & 4 & - & - & 3 & - & 8 \\
\hline Stenus juno & - & - & - & 1 & 30 & - & 6 & 3 \\
\hline Stenus nigritulus & - & - & - & - & - & - & 3 & - \\
\hline Stenus nitens & - & - & - & - & 3 & - & - & - \\
\hline Stenus pallipes & - & - & - & 5 & 3 & - & 22 & - \\
\hline Stenus palustris & - & - & - & - & - & - & 1 & - \\
\hline Stenus pusillus & - & - & 2 & - & 2 & - & - & - \\
\hline Stenus solutus & - & - & - & 1 & - & - & - & - \\
\hline Tachinus corticinus & - & - & 1 & - & 1 & - & - & - \\
\hline Tachinus signatus & 1 & - & - & 3 & 7 & 2 & - & 1 \\
\hline Tachyporus atriceps & - & 2 & - & 1 & - & - & - & - \\
\hline Tachyporus chrysomelinus & - & - & - & 1 & - & - & - & - \\
\hline Tachyporus dispar & 1 & 4 & 1 & 1 & - & 34 & 2 & 2 \\
\hline Tachyporus hypnorum & - & 4 & - & 2 & - & 1 & 2 & 6 \\
\hline Tachyporus nitidulus & 3 & 44 & 12 & 2 & 4 & 110 & - & 36 \\
\hline Tachyporus obtusus & - & - & - & - & - & 1 & - & 1 \\
\hline
\end{tabular}


TABle 6: Continued.

\begin{tabular}{|c|c|c|c|c|c|c|c|c|}
\hline Species & Lindhof & Dänisch-Nienhof & Stohl & Stakendorf & Hohenfelde & Hubertsberg & Behrensdorf & Weißenhaus \\
\hline Tachyporus pusillus & - & - & - & - & 1 & 1 & - & - \\
\hline Tachyporus quadriscopulatus & - & - & 1 & - & - & - & - & - \\
\hline Tachyporus solutus & - & - & - & 2 & - & - & - & - \\
\hline Tasgius ater & - & - & - & - & - & 1 & - & - \\
\hline Tasgius compressus & - & 2 & - & - & - & - & - & - \\
\hline Tasgius melanarius & 1 & 5 & 1 & 3 & - & - & 1 & 1 \\
\hline Tasgius morsitans & 10 & 4 & 1 & 2 & 1 & 1 & - & 1 \\
\hline Tasgius winkleri & - & 5 & - & 1 & - & 1 & - & - \\
\hline Thinonoma atra & 一 & 一 & - & 1 & 1 & 一 & - & 3 \\
\hline Tinotus morio & - & - & - & - & - & - & 1 & - \\
\hline Xantholinus linearis & 1 & - & 1 & 一 & 2 & 3 & - & 5 \\
\hline Xantholinus longiventris & 3 & 1 & - & 3 & 7 & 3 & 1 & - \\
\hline Zyras limbatus & - & - & - & - & - & - & - & 2 \\
\hline
\end{tabular}

in both investigations. Factors connected with wooded or nonwooded situations and moisture conditions were mainly responsible for the different species compositions of the habitats in both studies. Moreover, in a similar analysis of the spider fauna from the same sites analysed in the present investigation, the wood situation and moisture content were the main environmental parameters responsible for the separation of spider assemblages [19].

In specific investigations of North Sea salt marshes, Schaefer [20] found two habitat types: one from $80 \mathrm{~cm}$ to $130 \mathrm{~cm}$ above NN and one from $20 \mathrm{~cm}$ to $60 \mathrm{~cm}$ above NN. Consequently, elevation and frequency of inundation were the most important environmental factors. This was also true for a comprehensive study of staphylind assemblages in Baltic Sea salt marshes that included sites from the states of Schleswig-Holstein (north-western Germany) and Vorpommern (north-eastern Germany) [9]. In the salt marshes, soil conditions were less important than in the investigations on the sandy islands of Lower Saxony and in the present study of the Baltic Sea coast habitats.

The vertical gradient from sea level to higher elevations certainly affects the staphylinid assemblages of beaches and primary dunes. There has been no increase in species richness of Staphylinidae found between sandy beaches and primary dunes. However, specific species occurred on beaches that were not found in primary dunes, for example, Polystomota grisea and Phytosus spinifer. In contrast to Staphylinidae, a significant increase of species richness from beaches to primary dunes was found for spiders $[7,19]$. Schaefer [7] also found an increase in species richness in the adjacent habitats of dunes and dry grassland, representing a gradient of increasing elevation. The decrease of species richness from higher elevated sites to lower elevated sites in coastal habitats was referred to the higher instability of habitats exposed to the sea [20]. This effect of instability was also supposedly responsible for the decrease of species richness of other organisms such as meiofauna and macrofauna $[21,22]$ and might also account for the elevation gradient of species richness in salt marshes [23]. Since the high-energy input by wind and waves combined with high erosion is greater on shingle beaches than on sandy beaches, the low species richness of shingle beaches might be referred to the effect of instability. Nevertheless, several species are adapted to such unstable habitats as could be shown for the aleocharine species, for example, Aleochara bipustulata, Polystomota grisea, and P. punctatella that all live as parasitoids in flies without host specificity except host size [24]. The first species occurs in many sandy habitats of the mainland, but the abundance decreases from high elevated dune sites to low elevated beach sites at coasts. On the other hand, the two other species are restricted to beach habitats only although host species are the same as in $A$. bipustulata. It can be assumed that the flies live off the rich wrack debris on beaches which exhibit rich food resources for parasitoid staphylinids and can compensate for the loss in species richness found in other animal groups.

When considering all investigated parameters, lagoons are certainly the most species-rich habitats for staphylinids in this study. However, sandy beaches also reveal high numbers of species, particularly the endangered species. 5 species on the red list of endangered species status 1 and 2 have been found on sandy beaches, whereas only 2 were found at lagoons. Moreover, total species richness on sandy beaches as estimated by the Jackknife II method is only slightly lower than at lagoons, but distinctly higher than in all other habitat types. The eminent status of sandy beaches becomes still more relevant concerning exclusive species for the habitat. According to this investigation, only one exclusive species has been found at lagoons, Stenus pallipes. However, this species is abundant at most lake margins in northern Germany. In comparison, 5 exclusive species have been found at beaches. Overall, beaches support a higher number of endangered species and a much higher number of exclusive species compared to lagoons. Therefore, from a regional point of view, sandy beaches are the hot spots of species richness.

Another habitat also seems to be of great value for the coastal species richness at the Baltic Sea: the woodless cliffs. Species richness is distinctly lower than on beaches, but many exclusive and rare species live there. In particular, Stenus fossulatus, Bledius defensus (RL, 2), and Anotylus insecatus 
seem to be adapted to open, bare soil habitats on clay or silt soils. The distribution of the two species A. insecatus and $B$. defensus also indicates that the specific mixtures of sand and silt material are responsible for the occurrence of these species. Regarding their occurrence in the sandsilt gradient, they prefer completely different mixtures of sand and silt/clay. B. defensus seems to be restricted to a very narrow range of the mixture gradient. Thus, not only the conservation of the cliff situation is needed but also the whole range of sand and silt mixtures is necessary to preserve habitats for the different demands of species.

The present results show that tourism significantly decreases species richness on sandy beaches. According to both species per trap and Jackknife II species richness, beaches open to tourists reveal approximately half of their potential species richness. The loss of species on beaches has been attributed to tourist activities for several species and animal groups. Unfortunately, this ecological problem has not been investigated at the species level in Germany. In South Africa, Moffett et al. [25] quantified the damage by tourist trampling for intertidal macrofauna and found a loss of $5 \%$ to $70 \%$ depending on the species. The negative effect of trampling on Talitrus saltator (Crustacea) has been determined in different studies [26-28]. According to their results, it is not possible to compensate for the losses during short periods of tourist activity during summer. The loss of the ground beetle Cicindela maritima to near extinction on Baltic Sea beaches has also been referred to tourist activity [29]. Discussions about this species considered not only the sensibility of larvae against trampling but also the large home range to be the cause for the high losses. The high sensibility of beach species to trampling can certainly be referred to the porous sandy soils. The existing instability of the sandy soils caused naturally by wind and waves increases dramatically if tourist trampling is added. It can be assumed that the increase of instability triggering the species loss from sandy to shingle beaches also causes the species loss from closed to open beaches. At present, no studies investigating the degree of loss under different intensities of tourist activity are available. According to Kammer and Schernewski [30], tourist activity fluctuates in relation to weather, season, and week day. In their study, they found tourist densities between $7 \mathrm{~m}^{2}$ person $^{-1}$ and $84 \mathrm{~m}^{2}$ person ${ }^{-1}$. However, no studies are available that provide information about the level of tourist density which can be tolerated while preserving the species richness of beaches. Nevertheless, the present study documents that beach conservation is needed in order to preserve the species richness of coasts, not only for birds.

\section{Appendix}

For more details, see Table 6 .

\section{References}

[1] J. Bohac, "Staphylinid beetles as bioindicators," Agriculture, Ecosystems \& Environment, vol. 74, no. 1-3, pp. 357-372, 1999.

[2] J. Klimaszewski, D. W. Langor, T. T. Work, J. H. E. Hammond, and K. Savard, "Smaller and more numerous harvesting gaps emulate natural forest disturbances: a biodiversity test case using rove beetles (Coleoptera, Staphylinidae)," Diversity and Distributions, vol. 14, no. 6, pp. 969-982, 2008.

[3] A. Rose, Räumliche und zeitliche Verteilungsmuster der Kurzflügelkäfer (Coleoptera, Staphylinidae) Nordsee-Düneninseln unterschiedlicher Sukzessionsstadien, Archiv zoologischer Publikationen, Martina Galunder-Verlag, Nümbrecht, Germany, 2001.

[4] U. Irmler and S. Guerlich, "What do rove beetles (Coleoptera: Staphylinidae) indicate for site conditions," FaunistischÖkologische Mitteilungen, vol. 8, pp. 439-455, 2007.

[5] M. F. Jedrzejczak, “The modern tourist's perception of the beach: is the sandy beach a place of conflict between tourism and biodiversity?" Coastline Reports, vol. 2, pp. 109-119, 2004.

[6] U. Riecken, P. Finck, U. Raths, E. Schroeder, and A. Ssymank, Rote Liste der gefährdeten Biotoptypen Deutschlands. Zweite fortgeschriebene Fassung 2006, Bundesamt für Naturschutz, Bonn, Germany, 2006.

[7] M. Schaefer, "Einfluss der raumstruktur in landschaften der meeresküste auf das verteilungsmuster der tierwelt," Zoologische Jahrbücher Systematik, vol. 97, pp. 55-124, 1970.

[8] U. Irmler, K. Heller, H. Meyer, and H. D. Reinke, "Zonation of ground beetles (Coleoptera: Carabidae) and spiders (Araneida) in salt marshes at the North and the Baltic sea and the impact of the predicted sea level increase," Biodiversity and Conservation, vol. 11, no. 7, pp. 1129-1147, 2002.

[9] S. Hennicke, Die ökologische Charakterisierung der epigäischen Kurzflüglerzönosen (Coleoptera, Staphylinidae) der Küstenüberflutungsmoore des Greifswalder Boddens, Dissertation, Universität Greifswald, 2007.

[10] J. Speybroek, D. Bonte, W. Courtens et al., "The Belgian sandy beach ecosystem: a review," Marine Ecology, vol. 29, supplement 1, pp. 171-185, 2008.

[11] J. H. Frank and K.-J. Ahn, "Coastal Staphylinidae (Coleoptera): a worldwide checklist, biogeography and natural history," ZooKeys, vol. 107, pp. 1-98, 2011.

[12] E. Schlichting, H.-P. Blume, and K. Stahr, Bodenkundliches Praktikum, Blackwell Science, Berlin, Germany, 1995.

[13] StatSoft Inc., STATISTICA für Windows [Software-System für Datenanalyse] Version, Hamburg, Germany, 2004, http://www .statsoft.com/.

[14] Ø. Hammer, D. A. T. Harper, and P. D. Ryan, "Past: paleontological statistics software package for education and data analysis," Palaeontologia Electronica, vol. 4, no. 1, 2001.

[15] D. S. Schoeman, R. Nel, and A. Goulart Soares, "Measuring species richness on sandy beach transects: extrapolative estimators and their implications for sampling effort," Marine Ecology, vol. 29, supplement 1, pp. 134-149, 2008.

[16] C. J. F. ter Braak and P. Smilauer, Canoco for Windows. Version 4.0, Centre for Biometry, Wageningen, The Netherlands, 1998.

[17] C. J. F. ter Braak, "Ordination," in Data analysis in Community and Landscape Ecology, R. H. G. Jongman, C. J. F. ter Braak, and O. F. R. van Tongeren, Eds., Pudoc, Wageningen, The Netherlands, 1987.

[18] W. Ziegler and R. Suikat, Rote Liste der in Schleswig-Holstein Gefährdeten Käferarten, Landesamt für Naturschutz und Landschaftspflege Schleswig-Holstein, Kiel, Germany, 1994.

[19] S. Vahder and U. Irmler, "The spider fauna of Baltic Sea coast habitats," Faunistisch-Ökologische Mitteilungen, vol. 9, pp. 131-148, 2010.

[20] M. Schaefer, "Auswirkung natürlicher und experimenteller Störungen in Grenzzonen von Ökosystemen, untersucht am Beispiel der epigäischen Arthropodenfauna," Pedobiologia, vol. 14, pp. 51-60, 1974. 
[21] L. Kotwicki, M. De Troch, B. Urban-Malinga, T. Gheskiere, and J. M. Weslawski, "Horizontal and vertical distribution of meiofauna on sandy beaches of the North Sea (The Netherlands, Belgium, France)," Helgoländ Marine Research, vol. 59, no. 4, pp. 255-264, 2005.

[22] E. Jaramillo and A. McLachlan, "Community and population responses of the macroinfauna to physical factors over a range of exposed sandy beaches in south-central Chile," Estuarine, Coastal and Shelf Science, vol. 37, no. 6, pp. 615-624, 1993.

[23] U. Irmler and K. Heller, "Zonierung der Staphylinidae in einem Salzgrünland der schleswig-holsteinischen Nordseeküste," Faunistisch-Ökologische Mitteilungen, vol. 8, pp. 219-229, 2002.

[24] C. Maus, B. Mittmann, and K. Peschke, "Host records of Parasitoid Aleochara Gravenhorst species (Coleoptera, Staphylinidae) attacking puparia of Cyclorrhapeous Diptera," Deutsche Entomologische Zeitschrift, vol. 45, pp. 231-254, 1998.

[25] M. D. Moffett, A. McLachlan, P. E. D. Winter, and A. M. C. De Ruyck, "Impact of trampling on sandy beach macrofauna," Journal of Coastal Conservation, vol. 4, no. 1, pp. 87-90, 1998.

[26] S. Barca-Bravo, M. J. Servia, F. Cobo, and M. A. Gonzalez, "The effect of human use of sandy beaches on developmental stability of Talitrus saltator (Montagu, 1808) (Crustacea, Amphipoda). A study on fluctuating asymmetry," Marine Ecology, vol. 29, supplement 1, pp. 91-98, 2008.

[27] V. G. Veloso, G. Nieven, M. Lozano et al., "Responses of talitrus amphipods to a gradient of recreational pressure caused by beach urbanization," Marine Ecology, vol. 29, supplement 1, pp. 126-133, 2008.

[28] J. M. Weslawski, A. Stanek, A. Siewert, and N. Beer, "The sandhopper (Talitrus saltator, Montagu 1808) on the Polish Baltic Coast. Is it a victim of increased tourism?" Oceanological Studies, vol. 29, no. 1, pp. 77-87, 2000.

[29] U. Irmler, "Population size and mobility of Cicindela maritima Dejean, 1822 (Coleoptera: Carabidae)," Angewandte Carabidologie, vol. 9, pp. 1-6, 2010.

[30] M. Kammler and G. Schernweski, "Spatial and temporal analysis of beach tourism using webcams and aerial photographs," Coastline Reports, vol. 2, pp. 121-128, 2004. 

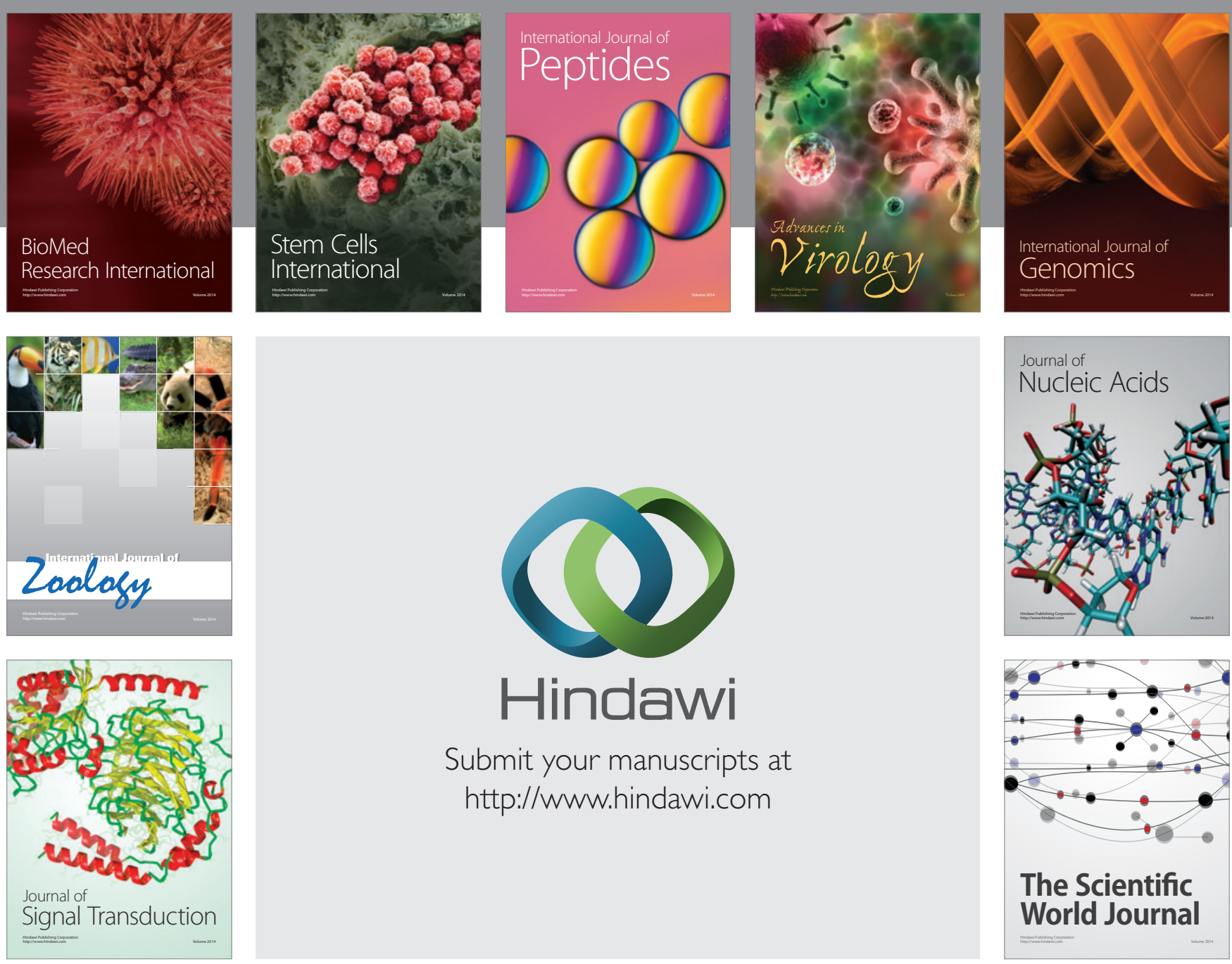

Submit your manuscripts at

http://www.hindawi.com
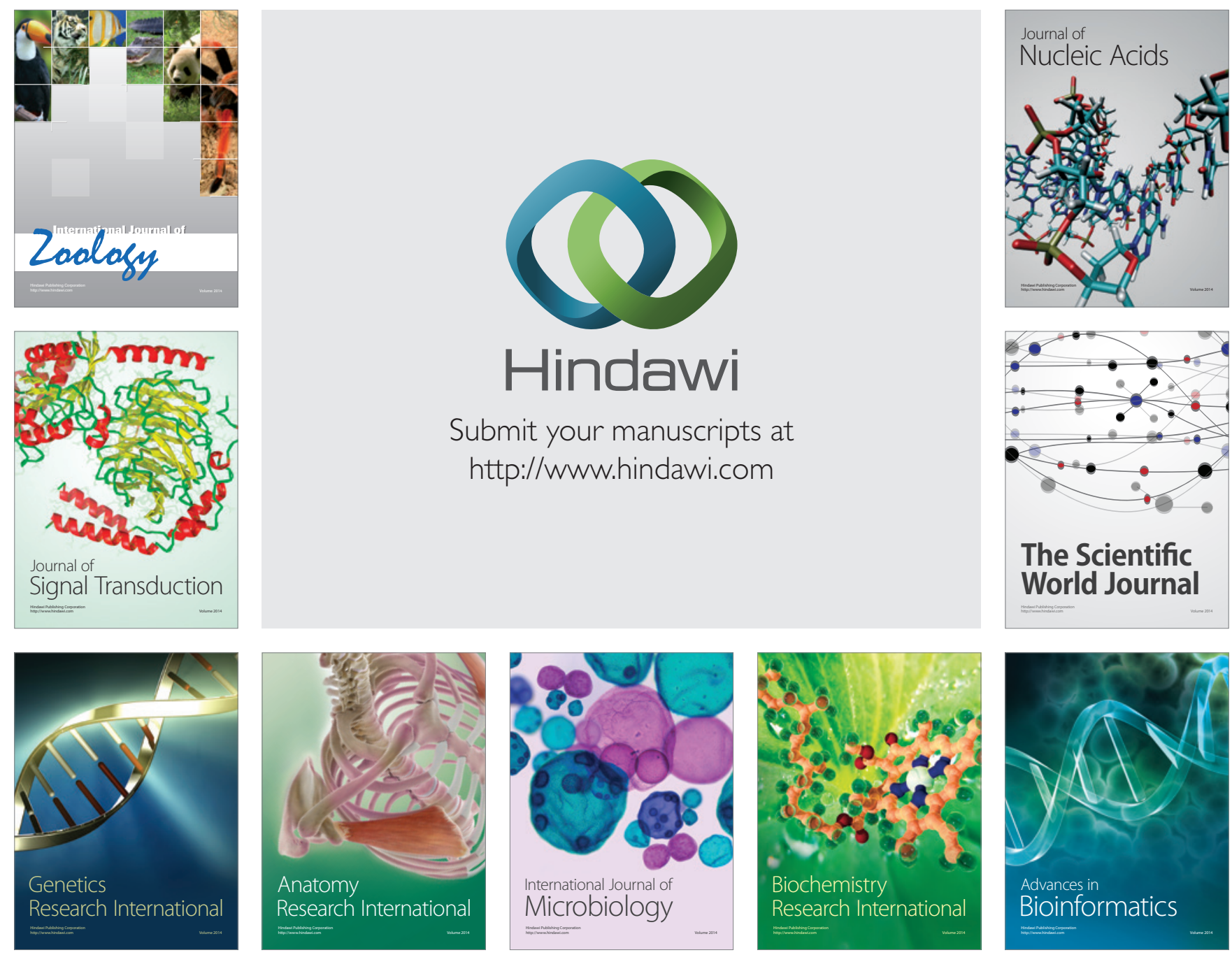

The Scientific World Journal
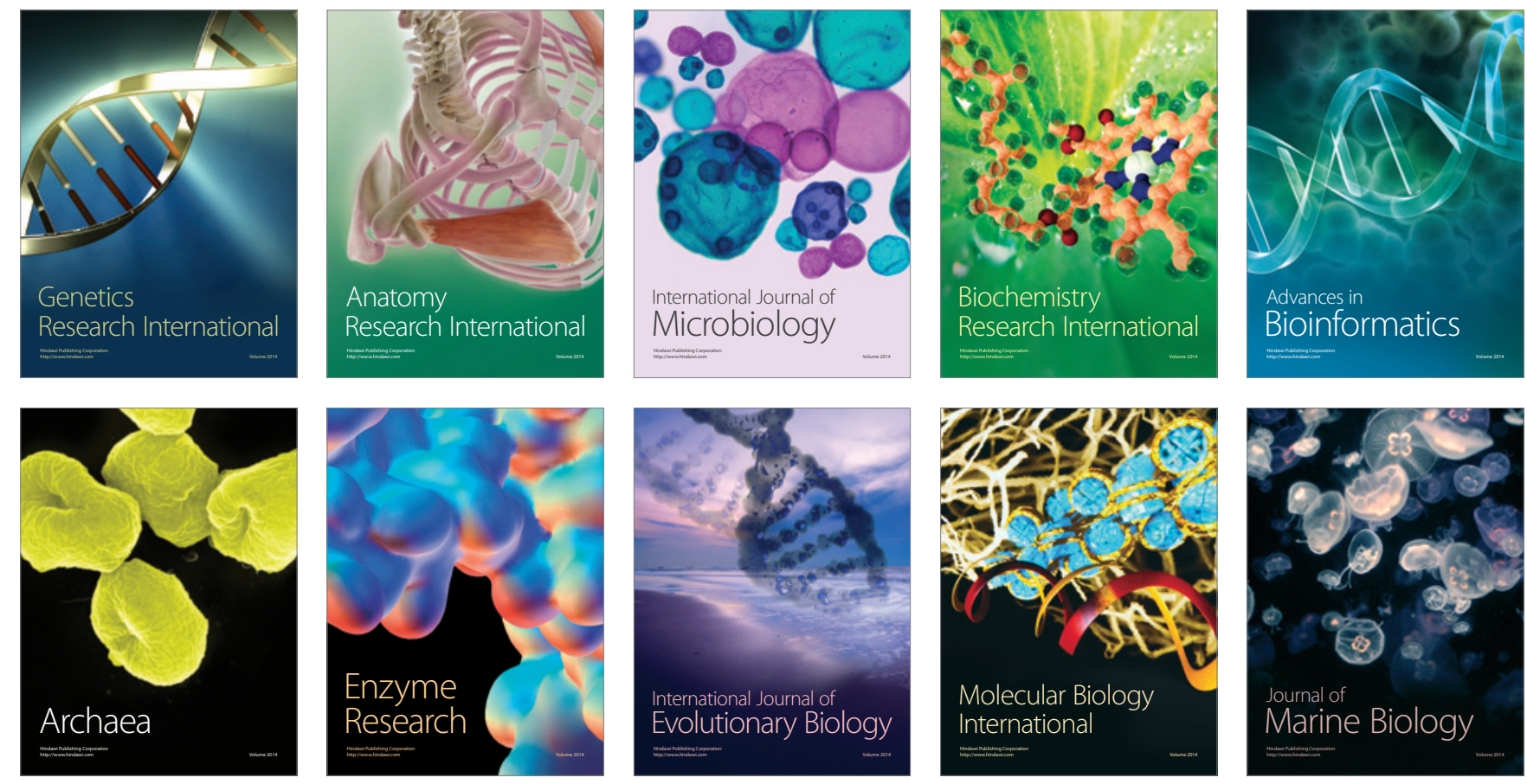